\title{
UNA CARACTERIZACIÓN DEL LENGUAJE METAFÓRICO DOCENTE Y SU DIVERGENCIA CON EL ENTENDIMIENTO DE LOS ESTUDIANTES RESPECTO DEL CONCEPTO DE NÚMERO RACIONAL'
}

A CHARACTERIZATION OF THE TEACHER'S METAPHORICAL LANGUAGE

AND ITS DIVERGENCE WITH THE STUDENT UNDERSTANDS ABOUT THE

RATIONAL NUMBER CONCEPT

Por: : Oscar Fernández Sánchez ${ }^{2}$

Cristian David Franco Restrepo ${ }^{3}$

Recibido: 24 de mayo de 2019 - Aprobado: 16 de octubre 2019

\section{RESUMEN}

Este artículo muestra los resultados de una investigación de tipo cualitativo-interpretativo en la cual se indaga sobre el discurso de dos docentes, con el fin de caracterizar el tipo de lenguaje metafórico utilizado en el aula para la enseñanza de los números racionales y la posible divergencia entre este lenguaje y el entendimiento de los estudiantes, causada por las expresiones metafóricas que utiliza el profesor en lenguaje no formal para explicar este tema a estudiantes de grado séptimo. Para el análisis del lenguaje metafórico de los docentes, se tiene en cuenta la teoría de la metáfora conceptual que aparece en Lakoff y Johnson, (1995).

Palabras clave: lenguaje metafórico, metáfora conceptual, enseñanza de los números racionales, discurso docente.

\footnotetext{
ABSTRACT

This article shows the results of a qualitative-interpretative research in order to characterize the type of metaphorical language used by two teachers in the classroom for the teaching of rational numbers. Moreover, the possible divergence between this language

${ }^{1}$ Producto derivado del proyecto de investigación Imaginarios matemáticos en el Eje Cafetero 2016-2017. Fase uno, Código 3-169, financiado por la Vicerrectoría de Investigaciones, Innovación y Extensión de la Universidad Tecnológica de Pereira.

${ }^{2}$ Profesor Titular de planta en la Facultad de Ciencias Básicas, Departamento de Matemáticas, Universidad Tecnológica de Pereira. Licenciado en Educación en la Especialidad Matemáticas, Universidad del Cauca, Magister en Ciencias Matemáticas, Universidad del Valle. Doctor en Ciencias de la Educación, RUDECOLOMBIA-UTP. Líder del Grupo de Investigación en Pensamiento Matemático y Comunicación-GIPEMAC. http://orcid.org/0000-0003-0804-2996

${ }^{3}$ Profesor nombrado en la Institución Educativa Nazario Restrepo, Secretaría de Educación de Caldas; docente catedrático en la Facultad de Ciencias Básicas, Departamento de Física, Universidad Tecnológica de Pereira. Licenciado en Matemáticas y Física. Magister en Enseñanza de la Matemática, Universidad Tecnológica de Pereira. Investigador del Grupo de Investigación en Pensamiento Matemático y Comunicación-GIPEMAC. Correo electrónico: cdfranco@utp.edu.co
} 
Oscar Fernández Sánchez - Cristian David Franco Restrepo

and the students' understanding caused by the metaphorical expressions used in non-formal language to explain that topic to seventh grade students. To analyze the teachers' metaphorical language, the conceptual metaphor theory of Lakoff \& Johnson, 1995 was used.

Keywords: Metaphorical language, conceptual metaphor, teaching of the rational numbers, teacher discourse.

\section{INTRODUCCIÓN}

El lenguaje es un aspecto fundamental en la comunicación en el aula de clase, en la cual están presentes el discurso tanto del docente como de los estudiantes, así como el de los autores de los textos que eventualmente el docente utilice. Dichos discursos están estructurados desde el imaginario social de los actores que los usan, en tanto miembros de un colectivo social, un imaginario que es posible rastrear por las metáforas que ellos utilizan, en la mayoría de las veces, de manera inconsciente; un hecho que es resaltado por Lizcano, (2006) y caracterizado por Lakoff y Johnson, (1995) en el lenguaje coloquial. Aunque, según Lakoff y Nuñez, (2000), el lenguaje metafórico no solo está presente en el lenguaje coloquial, sino también en el lenguaje especializado como el de la Matemática.

El docente juega un papel fundamental en la enseñanza de las matemáticas, por medio de sus competencias transmite sus saberes de acuerdo a los contenidos requeridos; una de las herramientas fundamentales para el desarrollo de sus cursos, es el lenguaje; con el cual se desarrolla a través de la elaboración, argumentación, interpretación de ideas que se transmiten por un canal de mensajes nutrido de expresiones metafóricas en su mayoría no planificadas. Una de las problemáticas por la que los estudiantes no desarrollan su conocimiento, es el escaso entendimiento del lenguaje utilizado por el docente, como se pudo comprobar con el desarrollo de la investigación que aquí se presenta.

A la luz de la teoría señalada se hizo una indagación sobre el nivel de divergencia entre el lenguaje utilizado por dos profesores para la enseñanza de los números racionales y el entendimiento por parte de los estudiantes. Así mismo, con los datos obtenidos se hizo una caracterización, expresada mediante gráficos relacionales, de las metáforas que usaron los profesores en sus clases para enseñar el tema mencionado antes. Las dos búsquedas se hicieron con estudiantes de grado séptimo de la Institución Educativa Jaime Salazar Robledo de la ciudad de Pereira. Con esta indagación se esperaba responder a las siguientes cuestiones: Primera: ¿Cuál es el nivel de coincidencia entre el discurso de aula de los dos profesores cuando enseñan el tema números racionales y el entendimiento de los estudiantes de séptimo de la Institución Educativa Jaime Salazar Robledo de Pereira?, y segunda: ¿Cómo se caracteriza el discurso metafórico de cada profesor cuando enseña el tema números racionales a estudiantes de grado séptimo de la Institución Educativa Jaime Salazar Robledo de Pereira?

Para esta investigación se tuvo en cuenta, principalmente, los criterios teóricos sobre metáforas 
conceptuales de Lakoff y Johnson, (1995), resultados de investigación en Font y Acevedo, (2003) y los obtenidos en el grupo de investigación GIPEMAC, en cuanto al lenguaje metafórico docente. Posteriormente se realizó el trabajo de campo donde se grabó el audio de las clases de los docentes investigados, luego se hicieron las transcripciones de los audios de las clases de cada profesor, con estas se procedió a hacer la codificación abierta del corpus de metáforas de cada docente, luego se hizo la codificación axial y selectiva sugerida en Strauss y Corbin, (2002), logrando así la categorización que permitió realizar los contrastes entre el discurso de los docentes y el entendimiento de los estudiantes.

Para la realización del contraste, se diseñan dos encuestas basadas en el corpus de metáforas encontrado, una para los estudiantes donde se les indagó sobre que entendieron acerca las expresiones metafóricas usadas por el docente en la clase desarrollada días anteriores, y a los docentes se les pregunta acerca de su intención en el uso de las expresiones metafóricas seleccionadas. Para la sistematización de los resultados, se utiliza el referente de análisis de la escala tipo Likert, con el fin de establecer el grado de coincidencia o divergencia entre los dos aspectos comunicativos presentes en el aula, el discurso del docente y el entendimiento de éste por parte de los estudiantes.

\section{Marco teórico}

\section{Teoría de la metáfora conceptual}

Esta es una teoría propuesta por Lakoff y Núñez, (2000) en la que afirman que las ideas presentes en el lenguaje de la Matemática surgen de los mecanismos cognitivos y corporales de las personas, por razones de tipo evolutivo, sociales, culturales y de relaciones con el entorno.

Según Font y Acevedo, (2003: 407), "la naturaleza de las matemáticas hay que buscarla en las ideas de las personas, más no en las demostraciones y rigurosidades propias de las matemáticas". Según estos mismos autores recientemente, la ciencia cognitiva no dualista en la que se basa la propuesta de Lakoff y Nuñez, (2000), ha realizado importantes avances en la comprensión del funcionamiento de la mente y más en concreto en lo que atañe a nuestra comprensión de las matemáticas. Esto se puede apreciar en los siguientes apuntes:

1) La importancia que tiene el cuerpo sobre la mente. La naturaleza y dinámica de nuestros cuerpos, cerebros y el funcionamiento de todos los días tienen una importancia fundamental en la estructura de la razón humana, la cual incluye el pensamiento matemático. 2) El papel del conocimiento inconsciente. La mayoría de los procesos cognitivos son inconscientes, en el sentido de que no son accesibles a nuestra introspección consciente, puesto que no podemos llegar directamente a nuestros sistemas conceptuales y a nuestros procesos cognitivos de nivel inferior que incluyen una gran parte del pensamiento matemático. 3) El pensamiento metafórico. La mayor parte de los seres humanos conceptúan conceptos abstractos en términos de objetos concretos y usan la estructura inferencial y maneras de razonar conectadas con nuestro sistema motor y sensorial. El mecanismo cognitivo que permite que lo abstracto se comprenda en términos de lo concreto es la metáfora conceptual. Lakoff y Núñez, (2000: 407-408). 


\section{Pensamiento metafórico}

Según Font y Acevedo, (2003), las metáforas se caracterizan por crear un puente conceptual entre el dominio de partida y el dominio de llegada, donde el dominio de partida hace referencia a la metáfora utilizada en el discurso y el dominio de llegada el significado pretendido por el docente. Dicen estos autores que otra de las funciones que cumple la metáfora es la de enlazar diferentes sentidos $\mathrm{y}$, en consecuencia, ampliar el significado que tiene para una persona un determinado objeto matemático, incluso para comprenderlo desde otra perspectiva.

A través de diversas apreciaciones e investigaciones en didáctica de la matemática, el pensamiento metafórico revela grandes implicaciones en la construcción del significado de los objetos matemáticos. Aunque aquello que ayuda puede constituirse en un obstáculo como advierte, Lakoff y Nuñez, (2000), debido a que las metáforas también llevan asociada cierta confusión y aparentes paradojas si estas no están claramente definidas o se interpretan de manera literal.

\section{Metáfora conceptual}

Soriano, (2012: 87), considera que "la metáfora conceptual es un fenómeno de cognición en el que un área semántica o dominio se representa conceptualmente en términos de otro". Se utiliza generalmente un concepto concreto para representar otro abstracto, que es lo que ocurre en el aula de clase de matemáticas, donde el docente se ve en la necesidad de utilizar dominios conceptuales concretos a través de ejemplos cotidianos para los estudiantes, para tratar de explicar elementos de dominios conceptuales abstractos.

En este sentido, un concepto se corporiza dependiendo de la interpretación que se realiza de este a través de la experiencia del individuo, originando tanto en el discurso del profesor como en el aprendizaje del estudiante una respuesta corporeizada.

Lakoff y Núñez, (2000), consideran que en el lenguaje de las matemáticas es posible distinguir tres tipos de metáforas, llamadas conceptuales, debido a que son utilizadas en el proceso de crear conceptos. Estas son las metáforas básicas o fundamentales, las cuales se presentan para fundamentar ideas matemáticas. Por ejemplo, "los números son puntos en una línea recta". Otro tipo de metáforas son las conectoras, con las cuales se vinculan dos campos conceptuales dentro de la misma matemática para hacer asequible un concepto. Por ejemplo, la metáfora "recta numérica"

conecta la Aritmética, en tanto números, con la Geometría en tanto la figura de la recta. Un tercer tipo son las metáforas de redefinición con las cuales se hace una sustitución de un concepto en términos no formales como "a sobre b" por la expresión "a divide a b", la cual es más técnica en el lenguaje de la Matemática.

\section{Metodología}

Se siguió un enfoque de investigación cualitativo-interpretativo, teniendo en cuenta como 
instrumento de investigación, sugerido por Hernández, Fernández y Baptista, (2006), la grabación de audios de las clases presenciales de dos docentes de grado séptimo de la Institución Educativa Jaime Salazar Robledo de Pereira, en la enseñanza de los números racionales. La variable de estudio fue la coincidencia entre las frases metafóricas del docente y el entendimiento de los estudiantes de la temática de las clases. Para la toma de datos se realizaron las transcripciones de los audios y luego mediante la técnica de Análisis de Contenido (AC) se procedió a realizar el análisis a nivel semántico de las expresiones del docente, para lo cual se tienen en cuenta las expresiones lingüísticas metafóricas como unidades de análisis para la indagación, como sugieren Navarro y Díaz, (1999). Siguiendo a Kerlinger, (1988: 543), "en lugar de observar el comportamiento de las personas en forma directa, o de pedirles que respondan a escalas, o aun de entrevistarlas, el investigador toma las comunicaciones que la gente ha producido y pregunta acerca de dichas comunicaciones" y en ese orden de ideas no se le pregunto directamente al docente sobre el uso de lenguaje metafórico en el desarrollo de sus clases, sino que se realizaron grabaciones de audio de sus clases, para posteriormente transcribirlas y con ellas se procedió a construir la codificación para generar las categorías según el marco teórico de la metáfora conceptual mencionada antes.

Se realizaron observaciones de clase de dos profesores, una de 46 minutos de clase con 30 estudiantes de grado séptimo-uno y para el segundo docente, se grabó un audio de 54 minutos, con 20 estudiantes de grado séptimo-dos, ambos en el año 2017. Luego de hacer los audios se procedió a hacer las transcripciones y a realizar el análisis de contenido para obtener un corpus de frases metafóricas. Con estas frases se elaboró un instrumento en forma de entrevista escrita para ser aplicada tanto a cada profesor como a los estudiantes en los dos grupos, con el fin de generar los datos con los cuales se hizo una confrontación entre la intención de los docentes cuando utilizan las frases metafóricas y el entendimiento de los estudiantes de dichas frases.

Aunque el interés en esta investigación no es analizar la influencia de factores socioeconómicos de los estudiantes ni de los docentes, tampoco aspectos como la formación de los docentes, vale la pena mencionarlos con el ánimo de que el lector se haga una idea acerca de posibles aspectos sociales que pudieran influir en los resultados que aquí se presentan y sirvan de insumos para motivar otras investigaciones en este sentido. En cuanto a la población de estudiantes, es mixta, presenta diversidad étnica, debido a la presencia de estudiantes afrodescendientes, indígenas de la etnia Embera-Chamí y mestizos. La población estudiantil proviene de la comuna Villa Santana de la ciudad de Pereira, la cual presenta serias problemáticas sociales que de alguna manera afecta el proceso educativo de los estudiantes. La edad de los estudiantes oscila entre los 12 y 15 años. Y en cuanto a los profesores, uno es de origen chocoano, Administrador Financiero de la Universidad del Tolima y el otro, es de la región del Eje Cafetero, Ingeniero Eléctrico de la Universidad Tecnológica de Pereira, ambos de género masculino.

A partir del análisis de las transcripciones de los audios de los dos profesores, teniendo en cuenta 
el marco teórico de la teoría cognitiva de la matemática y de la metáfora conceptual, se generaron las categorías y subcategorías. Estas se relacionan entre sí mediante un diagrama relacional como se ilustra en la Fig. 1, para el docente uno y en la Fig. 2, para el docente dos, atendiendo las sugerencias encontradas en Rojas, (2017).

Fig. 1. Esquema relacional del lenguaje metafórico del docente uno en la enseñanza de los números racionales

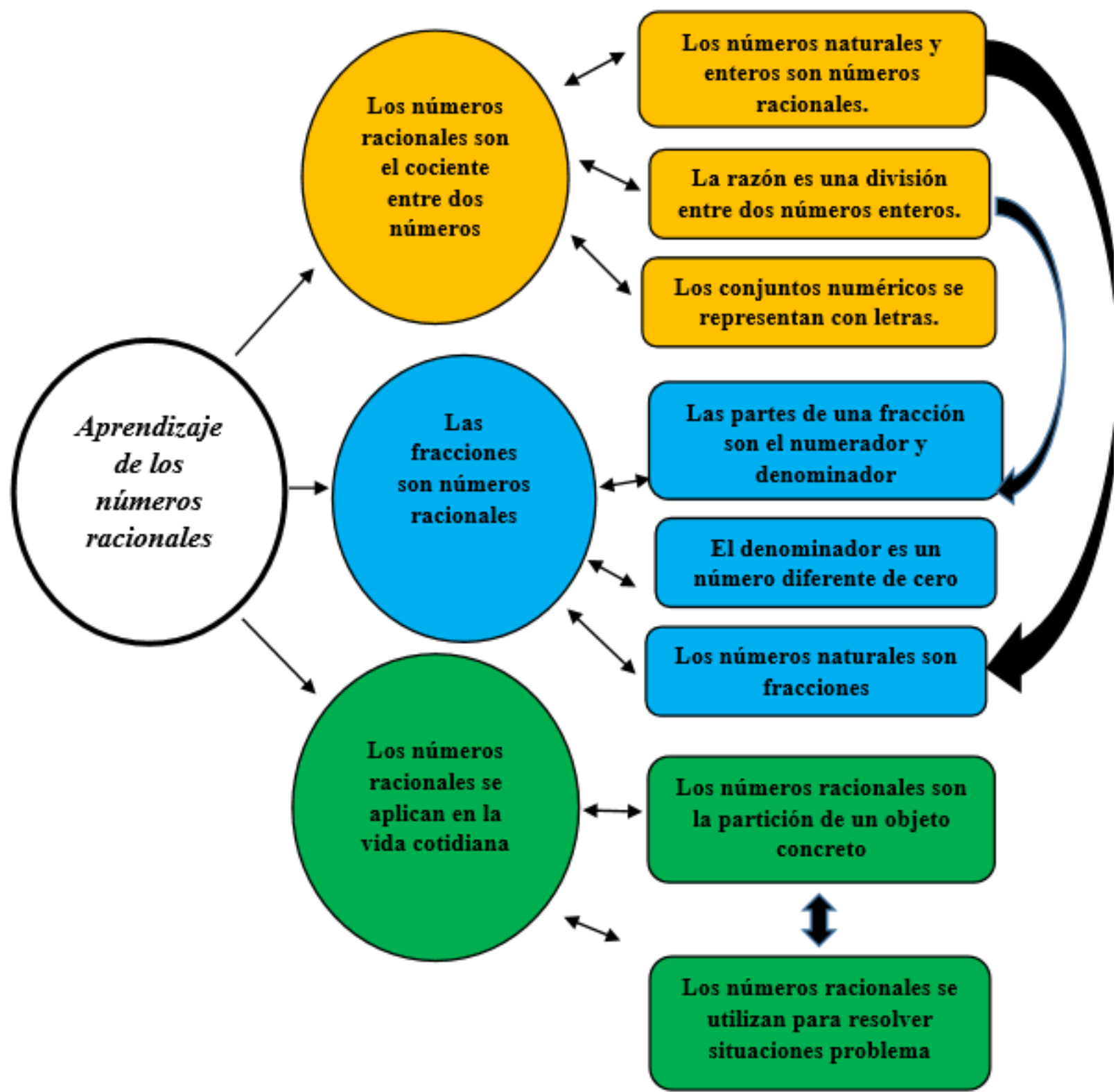

Fuente: Franco, (2018: 48) 
Fig. 2. Esquema relacional del lenguaje metafórico del docente dos en la enseñanza de los números racionales

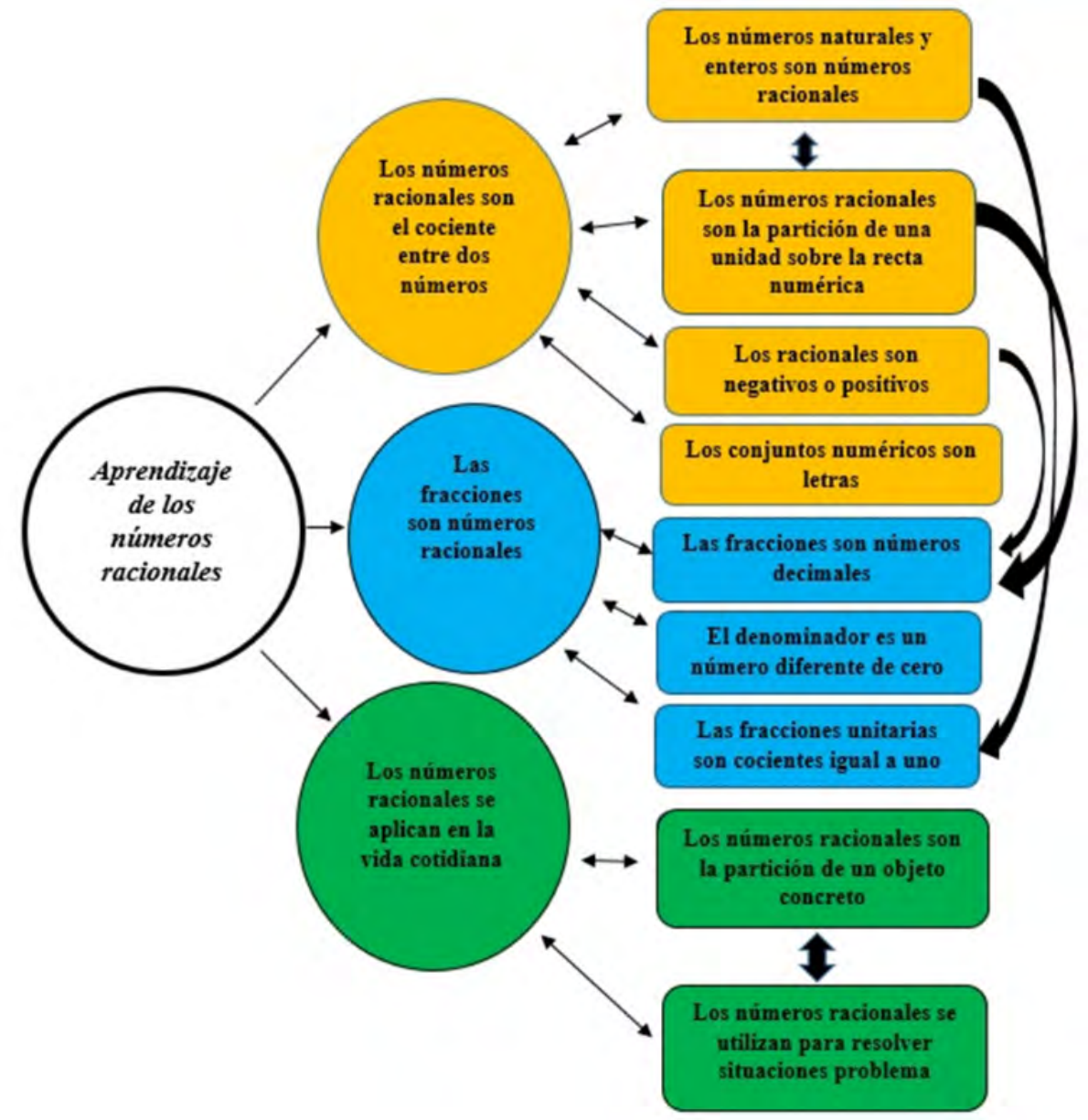

Fuente: (Franco, 2018: 49)

Para la cuantificación de la información, se asignan puntuaciones a las coincidencias según la escala de Likert sugerida en Hernández, et al., (2006), o método de evaluaciones sumativas, como también se le conoce. Las valoraciones de las respuestas de cada estudiante se suman para generar una puntuación total asignada a cada frase metafórica evaluada, para lo cual se construyó una tabla donde se relacionan las puntuaciones alcanzadas por cada estudiante en cada una de las frases metafóricas evaluadas mediante la aplicación del instrumento diseñado después del proceso de categorización.

\section{Variables estudiadas}

Se busca determinar el porcentaje de coincidencia entre el lenguaje metafórico empleado por los dos docentes frente al entendimiento de los estudiantes del grado séptimo, para el aprendizaje de los números racionales. Mediante análisis de contenido se hizo el análisis de las transcripciones de los audios y el resultado de la aplicación de cuestionarios diseñados por los investigadores tanto a los docentes como a los estudiantes. Con los resultados de las respuestas a dichos cuestionarios, se realizó una comparación entre las respuestas de cada estudiante a la pregunta por la comprensión 
de lo que expresó el docente a través de las frases metafóricas y el significado pretendido por el docente, con los resultados se determinó el porcentaje de coincidencia en las respuestas. Para la medición de dicha coincidencia, se utilizó la escala tipo Likert, la cual consiste en calificar de manera numérica una serie de ítems presentados en una encuesta con el fin de medir la variable de estudio.

Tabla 1. Calificación escala Likert

\begin{tabular}{|c|c|c|c|c|}
\hline Muy de acuerdo & De acuerdo & Ni de acuerdo, ni en desacuerdo & En desacuerdo & Muy en desacuerdo \\
\hline 5 & 4 & 3 & 2 & 1 \\
\hline
\end{tabular}

Fuente: Hernández, et al., (2006)

Según esta escala, si la respuesta del estudiante está muy de acuerdo con lo que pretendía el docente, se asigna una calificación de 5 , pero si lo expresado por el estudiante no coincide en nada con la pretensión por del docente, la calificación es 1, igual para los otros casos, como se puede ver en la Tabla 1.

Para este trabajo se consideró la variable coincidencia entre respuestas de los estudiantes frente a las del docente respecto al tema números racionales. Para ello, se le entregó el cuestionario a cada estudiante, para indagar por la comprensión de algunas expresiones metafóricas utilizadas en la clase de cada docente. De igual manera, a los docentes se les entregó un cuestionario para indagar sobre la intencionalidad al usar las expresiones metafóricas contenidas en frases metafóricas identificadas en las transcripciones de los audios de sus clases. Con la aplicación del cuestionario se obtuvo un corpus de frases metafóricas para los dos docentes (ver las Tablas 2 y 3 ).

Tabla 2. Corpus de frases metafóricas para el docente uno

\begin{tabular}{|l|l|}
\hline $\begin{array}{c}\text { Código de la frase me- } \\
\text { tafórica }\end{array}$ & \multicolumn{1}{|c|}{ Frase metafórica del docente } \\
\hline D1FM1 & Los números naturales los simbolizamos con la letra N, los números enteros con la Z mayúscula \\
\hline D1FM2 & $\begin{array}{l}\text { Como los números naturales se pueden representar con letras y números enteros se pueden representar } \\
\text { con la letra . }\end{array}$ \\
\hline D1FM3 & El 2 que es un entero y a la vez un número natural, lo puedo representar otra vez en forma de fracción. \\
\hline D1FM4 & $\begin{array}{l}\text { Este denominador como me decía Francisco no puede ser igual a cero, pues entonces, ya no será una } \\
\text { fracción. }\end{array}$ \\
\hline D1FM5 & $\begin{array}{l}\text { Los números racionales dijimos que los números racionales eran igual a lo siguiente: los números racio- } \\
\text { nales es igual a a/b donde a y b } \square \text { a los Z, pero b } \neq 0 \text { porque en una fracción no puede haber un denomi- } \\
\text { nador 0. }\end{array}$ \\
\hline D1FM6 & $\begin{array}{l}\text { Los que representamos para los números naturales, los utilizamos para representar situaciones las cuales } \\
\text { tenemos que mirar la relación entre dos cantidades. }\end{array}$ \\
\hline D1FM7 & Las relaciones que nosotros hacemos pueden ser positivas y negativas. \\
\hline D1FM8 & $\begin{array}{l}\text { En física la podemos utilizar para mirar desde cierta distancia recorriendo desde un automóvil desde } \\
\text { cierta distancia hasta la otra y miramos cúal será el tiempo recorrido para dicho recorrido. }\end{array}$ \\
\hline D1FM9 & $\begin{array}{l}\text { Si tenemos que hay un pastel que se divide en ocho partes iguales, ¿qué fracción representa esa parte del } \\
\text { pastel? }\end{array}$ \\
\hline
\end{tabular}




\begin{tabular}{|l|l|}
\hline D1FM10 & $\begin{array}{l}\text { En los números racionales las partes que se colorean se llaman numerador y en las que se divide se llama } \\
\text { denominador. Dijimos que aquí en esta relación, esta figura que estamos mostrando aquí se está dividien- } \\
\text { do, ¿en cuánto? }\end{array}$ \\
\hline D1FM11 & $\begin{array}{l}\text { Tenemos } 24 \text { naranjas y de esas 24, digamos que Yurleidy vino con hambre y dice comámonos } 12 \ldots \text { la } \\
\text { relación; las } 12 \text { naranjas son las que se come Yurleidy y las otras son las que están sobrando entonces } \\
\text { decimos es una relación. }\end{array}$ \\
\hline D1FM12 & Queda en 0, queda en la recta numérica en 0 ¿cierto?, queda en el punto de origen, el punto inicial. \\
\hline
\end{tabular}

Fuente: Elaboración de los autores

Tabla 3. Corpus de frases metafóricas para el docente dos

\begin{tabular}{|c|c|}
\hline $\begin{array}{l}\text { Código de la frase } \\
\text { metafórica }\end{array}$ & Frase metafórica del docente \\
\hline D1FM1 & $\begin{array}{l}\text { Los números enteros eran todos aquellos números que eran positivos y los negativos. Pero todos eran } \\
\text { enteros. }\end{array}$ \\
\hline D1FM2 & Los números enteros son aquellos que son de unidad en unidad. \\
\hline D1FM3 & $\begin{array}{l}\text { Decíamos que entre esos números también hay infinitos números, entre } 0 \text { y } 1 \text { hay infinitos números, entre } \\
1 \text { y } 2 \text { hay infinitos números, todos los que ustedes quieran. }\end{array}$ \\
\hline D1FM4 & $\begin{array}{l}\text { Cuando yo tengo una fracción donde el numerador y el denominador son iguales, decimos que son frac- } \\
\text { ciones unitarias. }\end{array}$ \\
\hline D1FM5 & $\begin{array}{l}\text { Los números racionales siempre tienen la particularidad de que se tienen que expresar como división } \\
\text { entre dos números. }\end{array}$ \\
\hline D1FM6 & $\begin{array}{l}\text { Entonces los fraccionarios vienen de ahí ¿Qué estamos diciendo? Que los números racionales son todos } \\
\text { los números, incluyendo también los números enteros, pero son todos los números que están en la mitad } \\
\text { de estos números también enteros, todo este conjunto es el conjunto de los números racionales. }\end{array}$ \\
\hline D1FM7 & $\begin{array}{l}\text { Estos números enteros son números racionales, porque todos los números enteros se pueden expresar } \\
\text { como la división de dos números, por ejemplo, el } 5 \text { puede ser, y el } 4 \text { puede ser, ya que } 12 \div 3 \text { igual a } 4 \text {, } \\
4 \text { lo puedo expresar también como. }\end{array}$ \\
\hline D1FM8 & $\begin{array}{l}\text { Las fracciones se pueden expresar de múltiples formas y muchas fracciones están expresando en reali- } \\
\text { dad la misma cantidad, esto lo denominamos fracciones equivalentes, que son aquellas que en cantidad } \\
\text { expresan lo mismo. }\end{array}$ \\
\hline D1FM9 & $\begin{array}{l}\text { Los fraccionarios a mí me sirven para representar también proporciones, cuando uno dice } 20 \text { de cada } 100 \\
\text { personas tienen los ojos verdes, entonces estamos diciendo que de } 100 \text { personas, solo } 20 \text { tienen los ojos } \\
\text { verdes. }\end{array}$ \\
\hline D1FM10 & $\begin{array}{l}\text { Entonces mire lo que sucede ahí, observen, ¿alguien trajo calculadora? Dividan } 10 \div 3 \text { en la calculadora, } \\
\text { ¿Cuánto le da?, eso da } 3,333 \ldots \text { resulta que este es un numero decimal periódico, porque mire que el nú- } \\
\text { mero } 3 \text { se empieza a repetir indefinidamente, o sea que este es un numero decimal periódico pero que se } \\
\text { repite infinitamente el } 3 \text {. Este es un número racional, es racional porque igual yo lo puedo expresar como } \\
\text { cualquier número que se exprese como la división entre dos números es un número racional, porque es } \\
\text { un número periódico. }\end{array}$ \\
\hline D1FM11 & $\begin{array}{l}\text { Si ustedes tienen una naranja y la dividen en cuatro cascos ¿cierto? En cuatro partes iguales, cada casqui- } \\
\text { to viene a ser , si se comen los cuatro cascos ¿me estoy comiendo cuantos cuartos?... es lo mismo que } \\
\text { tener una naranja. }\end{array}$ \\
\hline D1FM12 & $\begin{array}{l}\text { Si yo tengo tres mitades de naranjas, más dos mitades de naranjas, pues me da } 5 \text { mitades de naranjas, } \\
\text { entonces vamos a mirar eso ya rápidamente para finalizar. }\end{array}$ \\
\hline
\end{tabular}

Fuente: Elaboración de los autores

Para el análisis, la información se condensó en tablas, en la forma como se ilustra en la Tabla 4, la cual muestra una caracterización para las metáforas D1FM4 y D1FM11 identificadas en frases metafóricas presentes en el discurso del docente uno, así como la intención de ese docente respecto del uso de dichas frases. Así mismo, en la misma Tabla 4 se puede apreciar la caracterización expresada en la clasificación de las metáforas D2FM3 y D2FM6, identificadas en frases metafóricas 
presentes en el discurso del docente dos y su intención respecto del uso de dichas frases.

Tabla 4. Confrontación de frases metafóricas y la intencionalidad del docente

\begin{tabular}{|c|c|c|}
\hline Tipo y código de metáfora & Frase metafórica del docente & Intención del docente \\
\hline $\begin{array}{l}\text { Estructural-Básica-D1FM4 } \\
\text { Metáfora-estructura: Los números racio- } \\
\text { nales son el resultado de la división de un } \\
\text { número entero entre otro entero. }\end{array}$ & $\begin{array}{l}\text { "Este denominador como me decía } \\
\text { Francisco no puede ser igual a cero, } \\
\text { pues entonces, ya no será una frac- } \\
\text { ción" }\end{array}$ & $\begin{array}{l}\text { En una fracción el denominador me indi- } \\
\text { ca el número de partes iguales en que se } \\
\text { divide la unidad que se va a fraccionar, } \\
\text { teniendo en cuenta esto, si le colocamos } \\
\text { "0" como denominador no se fracciona- } \\
\text { ria y seguiría siendo un número entero. }\end{array}$ \\
\hline $\begin{array}{l}\text { Estructural-Básica-D1FM11 } \\
\text { Metáfora-estructura: Los números raciona- } \\
\text { les son una relación entre dos cantidades }\end{array}$ & $\begin{array}{l}\text { "Tenemos } 24 \text { naranjas y de esas } 24 \text {, } \\
\text { digamos que Yurleidy vino con ham- } \\
\text { bre y dice comámonos } 12 \ldots \text { la rela- } \\
\text { ción; las } 12 \text { naranjas son las que se } \\
\text { come Yurleidy y las otras son las que } \\
\text { están sobrando entonces decimos } \\
12 / 24 \text { es una relación." }\end{array}$ & $\begin{array}{l}\text { Explicar que varias cantidades las po- } \\
\text { demos utilizar como conjunto y que de } \\
\text { este conjunto nos representa fracciones } \\
\text { en este caso Yurleidy se comió la mitad } \\
\text { de las naranjas. }\end{array}$ \\
\hline $\begin{array}{l}\text { Estructural-Ontológica-D2FM3 } \\
\text { Metáfora-estructura: Los números raciona- } \\
\text { les son números que se ubican entre núme- } \\
\text { ros enteros } \\
\text { Metáfora-objeto: Los números racionales } \\
\text { son objetos que se ubican en la recta numé- } \\
\text { rica entre pares de enteros }\end{array}$ & $\begin{array}{l}\text { "Decíamos que entre esos números } \\
\text { también hay infinitos números, entre } \\
0 \text { y } 1 \text { hay infinitos números, entre } 1 \\
\text { y } 2 \text { hay infinitos números, todos los } \\
\text { que ustedes quieran." }\end{array}$ & $\begin{array}{l}\text { Cuando se termina de hacer el repaso } \\
\text { de los enteros, se pretende, que los es- } \\
\text { tudiantes, tengan en cuenta que entre } \\
\text { los enteros hay infinitos números que } \\
\text { corresponden a otro conjunto de núme- } \\
\text { ros llamados racionales, los cuales se } \\
\text { evidencian en la recta numérica dibujada } \\
\text { en el tablero. }\end{array}$ \\
\hline $\begin{array}{l}\text { Estructural-Básica-D2FM6 } \\
\text { Metáfora-estructura: Los números raciona- } \\
\text { les son todos los números }\end{array}$ & $\begin{array}{l}\text { "Entonces los fraccionarios vienen } \\
\text { de ahí ¿Qué estamos diciendo? Que } \\
\text { los números racionales son todos los } \\
\text { números, incluyendo también los nú- } \\
\text { meros enteros, pero son todos los nú- } \\
\text { meros que están en la mitad de estos } \\
\text { números también enteros, todo este } \\
\text { conjunto es el conjunto de los núme- } \\
\text { ros racionales." }\end{array}$ & $\begin{array}{l}\text { Se quiere explicar que el conjunto de los } \\
\text { números naturales y el conjunto de los } \\
\text { enteros están contenidos dentro del con- } \\
\text { junto de los racionales, ya que cualquier } \\
\text { natural o entero también puede ser ex- } \\
\text { presado como número fraccionario. }\end{array}$ \\
\hline
\end{tabular}

Fuente: Elaboración de los autores

Para realizar la medición con la escala tipo Likert, de la coincidencia entre la comprensión del estudiante de la frase metafórica empleada por el docente y la intención de este con el uso de dicha frase, se condensó la información usando el código de cada frase metafórica junto con la interpretación de los estudiantes de cada frase como se ilustra en la Tabla 5, en la cual se hace la comparación con la frase metafórica D1FM4, usada por el docente uno. De igual manera, en la Tabla 6, se presenta la comparación con la frase metafórica D2FM6, usada por el docente dos. 
Tabla 5. Calificación de coincidencia entre respuestas docente 1-estudiantes para la metáfora D1FM4

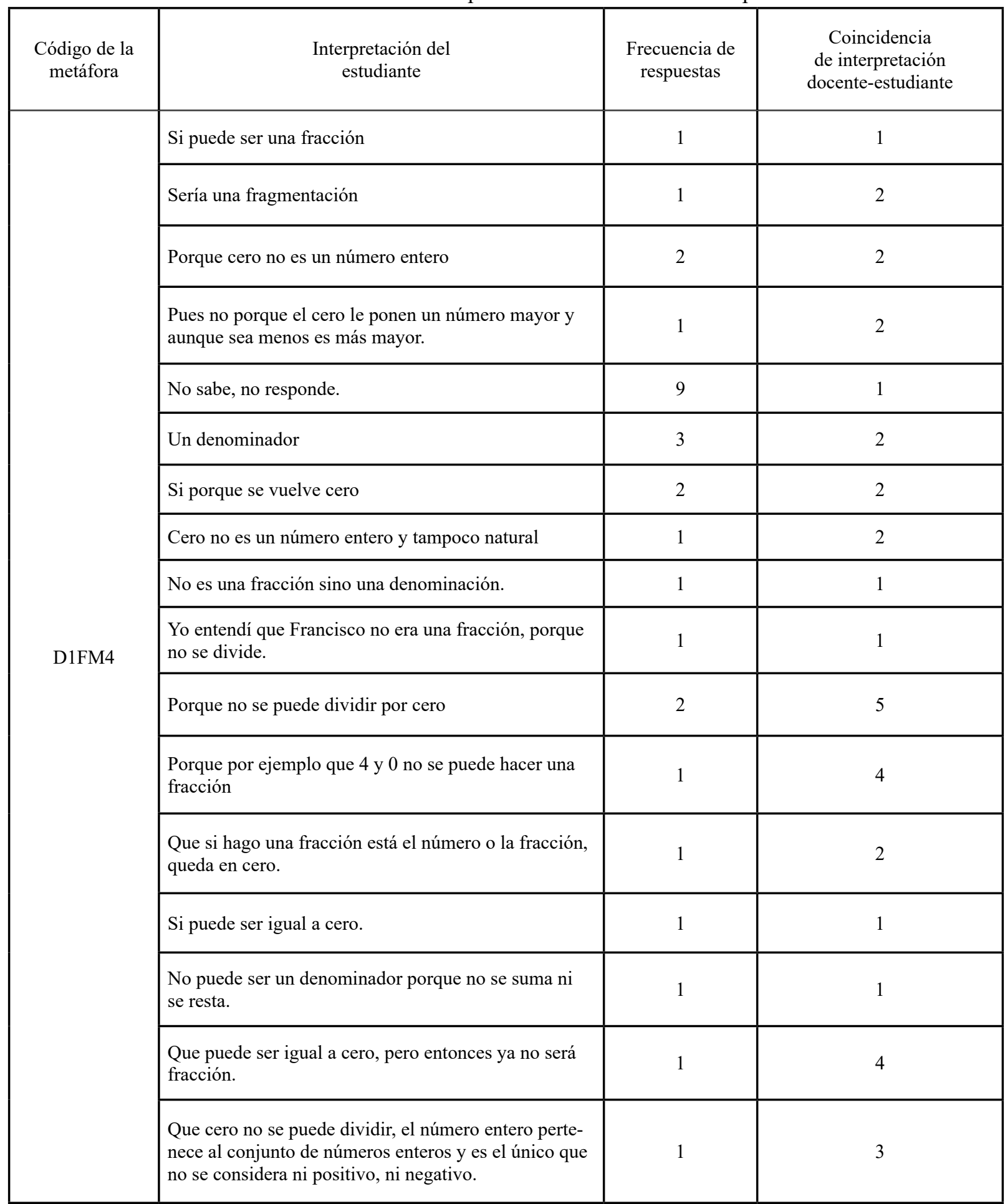

Fuente: (Franco, 2018: 113) 
Oscar Fernández Sánchez - Cristian David Franco Restrepo

Tabla 6. Calificación de coincidencia entre respuestas docente 2-estudiantes para la metáfora D2FM6

\begin{tabular}{|c|c|c|c|}
\hline $\begin{array}{l}\text { Código de la } \\
\text { metáfora }\end{array}$ & $\begin{array}{l}\text { Interpretación del } \\
\text { estudiante }\end{array}$ & $\begin{array}{l}\text { Frecuencia de } \\
\text { respuestas }\end{array}$ & $\begin{array}{l}\text { Coincidencia de interpretación } \\
\text { docente- estudiante }\end{array}$ \\
\hline \multirow{4}{*}{ D2FM6 } & No sabe no responde & 16 & 1 \\
\hline & $\begin{array}{l}\text { Que los números racionales también son } \\
\text { números enteros y los números que van en } \\
\text { la mitad también ej.: } 3.2-5.7 \text { y así porque se } \\
\text { dividen entre dos } 5 \div 2 \text { nos da } 2.5 \text { y eso sería un } \\
\text { numero racional. }\end{array}$ & 2 & 4 \\
\hline & $\begin{array}{l}\text { Los números racionales y los números enteros } \\
\text { no se dan con la misma operación. }\end{array}$ & 1 & 3 \\
\hline & $\begin{array}{l}\text { Todos los números son números racionales in- } \\
\text { cluyendo los enteros y los que están en la mitad } \\
\text { a esto se le denomina fracción equivalente. }\end{array}$ & 1 & 4 \\
\hline
\end{tabular}

Fuente: (Franco, 2018: 124)

\section{Resultados}

Se puede observar en la Tabla 4 que, la metáfora D1FM4 es de tipo estructural, puesto que la frase metafórica "Este denominador como me decía Francisco no puede ser igual a cero, pues entonces, ya no será una fracción”, está estructurada por la metáfora: "Los números racionales son el resultado de la división de un número entero entre otro entero”. Esta clasificación se hace según la tipología que sugiere Lakoff y Johnson, (1995).

Según los resultados que aparecen en la Tabla 5 se observa que esta frase metafórica afectó la forma como perciben el tema los estudiantes en el grupo del docente D1. Este hecho se verifica en las respuestas sobre la comprensión de la frase metafórica que utilizó el docente, puesto que primó la idea sobre el cero y los efectos sugeridos en la frase por el docente, para explicar la relación entre los enteros y la forma que adoptan los fraccionarios; de lo que se deduce que, el lenguaje formal institucionalizado, en términos de Brousseau, (2014), se ve afectado por el lenguaje coloquial presente en el discurso de aula del docente.

Fig. 3. Nivel de coincidencia entre las respuestas de los estudiantes con la intención del discurso del docente 1 (D1). Gráfico construido con Excel

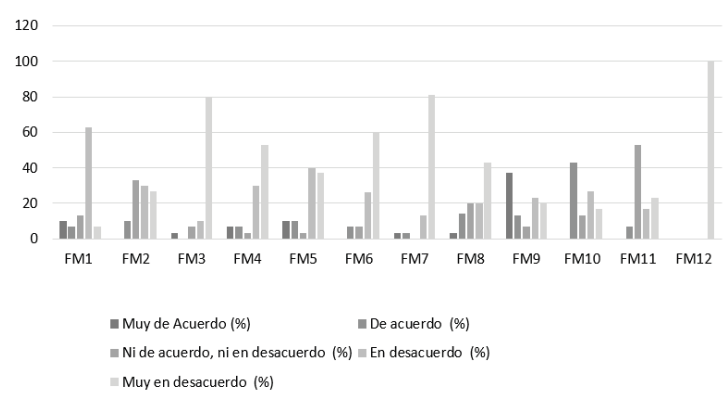

Fuente: (Franco, 2018: 54) 
Fig. 4. Nivel de coincidencia entre las respuestas de los estudiantes con la intención del discurso del docente 2 (D2). Gráfico construido con Excel.

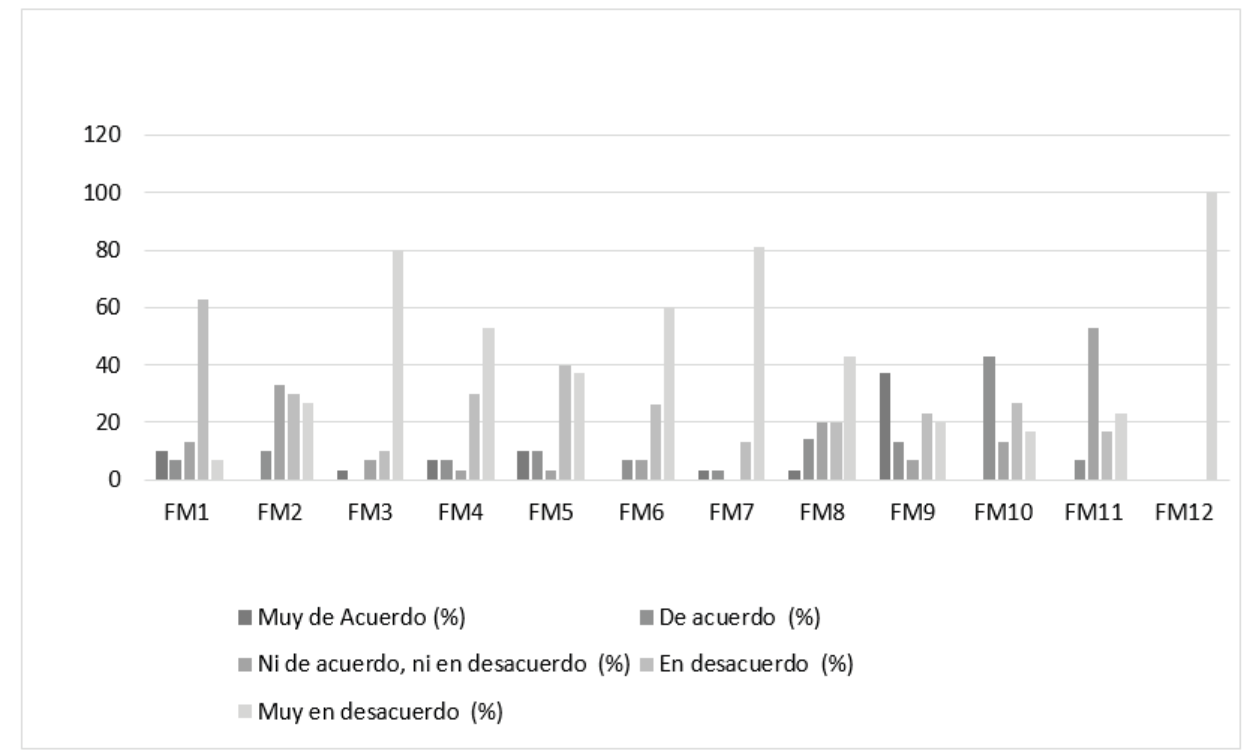

Fuente: (Franco, 2018: 57)

Las grabaciones del audio de las clases de los dos docentes, D1 y D2 se transcribieron y se aplicó la técnica del Análisis de Contenido a los textos obtenidos. Para el caso de los estudiantes en el grupo del docente D1, y la metáfora FM4 que aparece en la Tabla 5 y también en la Fig. 3, se verifican que el 6,67\% de las respuestas de los estudiantes están totalmente de acuerdo con la intención expresada por docente. En la misma Tabla 5, respecto a la misma frase metafórica D1FM4, también se puede apreciar que el 6,67\% están de acuerdo, aunque no totalmente, acorde con la escala Likert; el 3,33\% no están ni de acuerdo ni en desacuerdo, el 36,67\% están en desacuerdo y el 46,66\% están muy en desacuerdo. De estos porcentajes se puede inferir que esa frase metafórica utilizada en el discurso de aula por el docente D1, genera una divergencia entre lo que pretendía respecto al tema de números racionales y lo que realmente consiguió que los estudiantes le entendieran. Estos resultados sustentan el comentario hecho antes, sobre el hecho que el lenguaje coloquial primó sobre el lenguaje formal institucionalizado que el docente D1 pretendía enseñar. Ese hecho provocó que, en el imaginario de los estudiantes produjera ruido la idea del problema que implica el cero en el denominador cuando se desea establecer la relación entre los enteros y la forma fraccionaria de los números racionales. En el caso tratado aquí, se muestra como se sacrifica la generalidad implicada en el lenguaje formal institucionalizado, por el hecho particular expresado en la frase metafórica introducida por el docente D1.

Del análisis de contenido del discurso de aula del docente D1, se puede ver que este se caracterizó por la presencia de metáforas de tipo estructural principalmente, como se puede ver en la Tabla 4, debido a la presencia de metáforas estructurantes como: "Los números racionales son el resultado de la división de un número entero entre otro entero" con las cuales se enuncian frases metafóricas para 
explicar el concepto de número racional, con las implicaciones expuestas en la Tabla 5. Respecto al discurso de aula del docente D2, se puede apreciar en la Tabla 4 que se caracteriza por la presencia no solo de metáforas estructurales sino también ontológicas, en la medida que el docente utiliza frases metafóricas como: "Los números racionales son objetos que se ubican en la recta numérica entre pares de enteros" en la cual se refiere a los números racionales como objetos que se pueden manipular, como si fuesen objetos físicos.

\section{CONCLUSIONES}

En primera instancia se verifica que tanto el docente D1 como el docente D2 incorporan de manera inconsciente metáforas conceptuales en su discurso de aula, sobre todo metáforas estructurales y ontológicas, según aquellas caracterizadas por Lakoff y Johnson, (1995), es decir, se evidencia del análisis de contenido aplicado a las transcripciones de las clases de los dos docentes la presencia de metáforas estructurales, para el caso del docente D1, quien estructura sus explicaciones sobre el concepto de número racional mediante metáforas estructurales como: "Los números racionales son el resultado de la división de un número entero entre otro entero" o también "Los números racionales son una relación entre dos cantidades". Este hecho influenció la forma como los estudiantes comprendieron lo que sobre los números racionales, el docente pretendía enseñar. De manera análoga, el docente D2, estructura su discurso respecto a los números racionales usando metáforas estructurales y ontológicas. Para ilustrar, un ejemplo de metáforas ontológicas que usa este docente, la frase metafórica "Decíamos que entre esos números también hay infinitos números, entre $0 \mathrm{y} 1$ hay infinitos números, entre 1 y 2 hay infinitos números todos los que ustedes quieran", deja la sensación que "Los números racionales son objetos que se ubican en la recta numérica entre pares de enteros". Esto responde a la segunda pregunta planteada al comienzo del artículo, es decir, ¿cómo se caracteriza el discurso metafórico de cada profesor cuando enseña el tema números racionales a estudiantes de grado séptimo de la Institución Educativa Jaime Salazar Robledo de Pereira?

Y respecto a la primera pregunta ¿cuál es el nivel de coincidencia entre el discurso de aula de los dos profesores cuando enseñan el tema números racionales y el entendimiento de los estudiantes de séptimo de la Institución Educativa Jaime Salazar Robledo de Pereira? Se pudo evidenciar a través del análisis de contenido que la presencia de frases metafóricas genera, como se puede verificar de los resultados mostrados en la Fig. 2, para el docente uno y la Fig. 3, para el docente dos, que hay un alto grado de divergencia entre lo que pretendían los docentes D1 y D2, con las frases metafóricas que utilizaron en su discurso de aula cuando pretendieron explicar el concepto de número racional, frente al entendimiento de ese discurso que los estudiantes manifiestan. Por ejemplo, para el caso de la metáfora D1FM4, el hecho que el 6,67\% de las respuestas de los estudiantes está totalmente de acuerdo con la intención expresada por docente, el 3,33\% no están ni de acuerdo ni en desacuerdo, el 36,67\% están en desacuerdo y el 46,66\% están muy en desacuerdo, aunque no es el único caso, porque en el caso de las metáforas D1FM3, D1FM6, D1FM7 y D1FM12, más del 50\% de los estudiantes no comprendieron totalmente lo que el 
docente pretendía significar con el uso de las frases metafóricas usadas. Así mismo, para el caso del docente D2, se puede verificar ese mismo nivel de incomprensión para las metáforas D2FM6, D2FM10 y D2FM12.

\section{REFERENCIAS BIBLIOGRÁFICAS}

Brousseau, Guy. (2014). Fundamentos y métodos de la didáctica. En Trabajos de Enseñanza No. 5: Trad. Fregona, Dilma. Córdoba. Argentina: Universidad Nacional de Córdoba.

Franco, Cristian. (2018). Incidencia del lenguaje metafórico docente en el aprendizaje de los números racionales, en grado séptimo de la institución educativa Jaime Salazar Robledo de Pereira. Trabajo de grado de maestría. Pereira: Universidad Tecnológica de Pereira.

Font, Vicenç, Acevedo, Jorge. (2003). Fenómenos relacionados con el uso de metáforas en el discurso del profesor. El caso de las gráficas de funciones. En Enseñanza de las Ciencias, 21, 3, 405 418. Fecha de consulta 15 de junio de 2019 en: https://core.ac.uk/download/pdf/38990753.pdf

Hernández, Roberto, Fernández, Carlos y Baptista, Pilar. (2006). Metodología de la investigación. México, D.F.: McGraw Hill.

Kerlinger, Fred. (1988). Investigación del comportamiento. Tercera Edición. México DF.: Mc Graw Hill

Lakoff, George y Johnson, Mark. (1995). Metáforas de la vida cotidiana. Segunda Edición. Madrid: Cátedra. Fecha de consulta 2 de junio de 2019 en: https://linguisticaunlp.files.wordpress. com/2012/11/lakoff-y-johnson.pdf

Lakoff, George y NÚñez, Rafael. (2000). Where mathematics comes from: How the embodied mind brings mathematics into being. New York: Basic Books.

Lizcano, Emmanuel. (2006). Metáforas que nos piensan, sobre ciencia, democracia y otras poderosas ficciones. Ediciones Bajo Cero, bajo licencia de Creative Commons.

Navarro, Pablo y Díaz, Capitolina. (1999). Análisis de contenido. En DELGADO, Juan Manuel; y GUTIÉRREZ, Juan; (coordinadores): Métodos y técnicas cualitativas de investigación en ciencias sociales. Madrid: Síntesis.

Rojas, Claudia. (2017). Incidencia del lenguaje metafórico empleado por el docente en el aprendizaje del concepto de número complejo con estudiantes de grado noveno de la Institución Educativa Hogar Nazaret de Dosquebradas. Trabajo de grado de maestría. Pereira: Universidad Tecnológica de Pereira. 
Strauss, Anselm y Corbin, Juliet. (2002). Bases de la investigación cualitativa. Técnicas y procedimientos para desarrollar la teoría fundamentada. Trad. Eva Zimmerman. Medellín: Universidad de Antioquia. Fecha de consulta 8 de mayo de 2019 en https://diversidadlocal.files.wordpress. com/2012/09/bases-investigacion-cualitativa.pdf

Soriano, Claudio. (2012). La metáfora conceptual. En Ibarretxe, Iraide y Valenzuela, Javier. Lingüistica cognitiva. 87-109. Barcelona: Anthropos. 